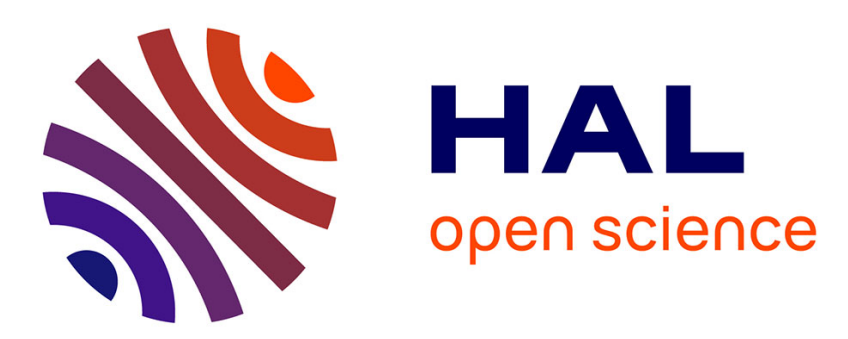

\title{
Charge dynamics through pi-stacked arrays of conjugated molecules: effect of dynamic disorder in different transport/transfer regimes
}

Alessandro Troisi

\section{- To cite this version:}

Alessandro Troisi. Charge dynamics through pi-stacked arrays of conjugated molecules: effect of dynamic disorder in different transport/transfer regimes. Molecular Simulation, 2006, 32 (09), pp.707716. 10.1080/08927020600857305 . hal-00514992

\section{HAL Id: hal-00514992 \\ https://hal.science/hal-00514992}

Submitted on 4 Sep 2010

HAL is a multi-disciplinary open access archive for the deposit and dissemination of scientific research documents, whether they are published or not. The documents may come from teaching and research institutions in France or abroad, or from public or private research centers.
L'archive ouverte pluridisciplinaire HAL, est destinée au dépôt et à la diffusion de documents scientifiques de niveau recherche, publiés ou non, émanant des établissements d'enseignement et de recherche français ou étrangers, des laboratoires publics ou privés. 


\section{Molecular Simulation}

Journal of

Experimental Nanoscience

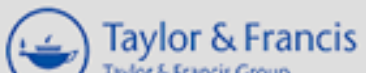

Taylor \& Francis Group

\section{Charge dynamics through pi-stacked arrays of conjugated molecules: effect of dynamic disorder in different transport/transfer regimes}

\begin{tabular}{|r|l|}
\hline Journal: & Molecular Simulation/Journal of Experimental Nanoscience \\
\hline Manuscript ID: & GMOS-2006-0065.R1 \\
\hline Journal: & Molecular Simulation \\
\hline $\begin{array}{r}\text { Date Submitted by the } \\
\text { Author: }\end{array}$ & 03-Jun-2006 \\
\hline Complete List of Authors: & Troisi, Alessandro; University of Warwick, Chemistry \\
\hline Keywords: & Charge Transfer, Organic Semiconductor, Quantum Dynamics \\
\hline
\end{tabular}

\section{SCHOLARONE ${ }^{m}$ \\ Manuscripts}


Charge dynamics through pi-stacked arrays of conjugated molecules: effect of dynamic disorder in different transport/transfer regimes

\author{
Alessandro Troisi \\ Department of Chemistry and Centre of Scientific Computing, \\ University of Warwick, CV4 7AL Coventry, UK
}

\begin{abstract}
We provide further computational evidence that the electronic coupling between pi-stacked molecules is strongly modulated by the thermal motions at room temperature, not only in supramolecular flexible systems (like DNA) but also in molecular crystals. The effect of this modulation on the charge dynamics is different for different transfer/transport mechanisms and depends on the modulation timescale. In the case of charge transfer between a donor and an acceptor, the effect of electronic coupling fluctuations introduces a corrective term in the expression of the rate constant (different for adiabatic and non-adiabatic charge transfer). For the transport in molecular crystals, this fluctuation can be the limiting factor for the charge mobility. Although the fluctuation of the electronic coupling is similar in magnitude for all systems containing molecular pi-stacking, its importance for the charge dynamics increases with the decrease of the reorganization energy.
\end{abstract}




\section{Introduction}

A large number of systems with useful and interesting electronic properties contain pi-stacked organic conjugated molecules. Molecular solids based on conjugated molecules constitute the most technologically relevant example, since they have been proposed as materials for the realization of large scale organic electronics devices. ${ }^{[1-3]}$ Also DNA, regarded as an electronic material, offers the interesting possibility of charge transport along its pi-stacked nucleobases and it received great attention for its potential role in self-assembling circuits. ${ }^{[4-6]}$ Other important classes of pi-stacked materials include discotic liquid crystals like hexabenzocoronene derivatives $\left.{ }^{[7,} 8\right]$, which form columnar stacks, and highly ordered polymers like poly(3-hexylthiphene) that found application in thin film transistors. ${ }^{[9]}$ The electronic structure of pi-stacked systems is largely determined by that of their molecular components since the energy splitting of the molecular levels is larger than the electronic coupling between the levels of close molecules. From a solid state perspective, one can say that the molecule itself defines the band gap while the intermolecular coupling defines the (always narrow) bandwidth. The weakness of the intermolecular interactions makes these materials quite different from inorganic semiconductors to which they have been often compared. It was noted many times ${ }^{[10]}$ that, because of the small interaction between molecules, organic materials are very soft and the coupling between the electronic and vibrational degrees of freedoms is expected to be very high. In this paper we investigate the role of nuclear motions in the modulation of the intermolecular electronic coupling (off diagonal electron-phonon coupling) with particular emphasis on the effect that this modulation produces on the charge dynamics. We aim at providing a broad perspective on the effect of these fluctuations in the different cases of interest to physical chemistry (from biophysics to material science) and we will attempt a classification of the possible phenomenologies.

The study of nuclear motions modulating the intermolecular coupling is an important computational problem with dramatic consequences on the theoretical modeling of the charge transport/transfer. In literature, the computational and theoretical studies are usually presented in different papers, while, in this contribution, we will stress the strong relation between the two aspects. In the next section, we describe a computational approach used to evaluate the 
effect of molecular motions on the intermolecular coupling. The similitude between the previously published results on DNA and the new computation on organic semiconductors will be used to attempt a generalization for all materials containing organic pi-stacked molecules. Section 3 will focus instead on the theoretical consequences of these results on the charge dynamics: different transport mechanisms will be assumed and the effect of the electronic coupling modulation will be considered for each of them.

\section{Fluctuation of the electronic coupling: computational assessment and some generalizations}

The electronic coupling between one-electron states localized on different molecules is not an experimental observable but can be evaluated computationally using a variety of techniques. Many researchers that investigated computationally the dependence of the coupling on various system parameters found it extremely dependent on the system geometry. ${ }^{[10,11]}$ One of the first systematic observations of this type was carried out by Kazmaier and Hoffmann ${ }^{[12]}$ who were able to prove, with simple extended Hückel calculations, that the different electronic spectrum (and color) of a family of perylene derivatives is due to the different electronic coupling between molecules in the crystal. The sensitivity of the electronic coupling to the system geometry can be an essential component of the electron dynamics if the conformational space explored at room temperature causes large fluctuations in the intermolecular electronic coupling.

DNA. To assess the role of electronic coupling fluctuation in DNA at room temperature a standard classical molecular dynamics (MD) simulation was carried out on several DNA fragments and the electronic coupling between nucleobases was computed for snapshots of the MD simulation separated by 0.01 ps. ${ }^{[13]}$ Assuming that the charge is carried by electron holes, which can be described by a linear combination of the HOMO orbitals of the nucleobases, one can write a Hamiltonian that includes the effect of intermolecular fluctuations as:

$$
H=\sum_{i} \varepsilon_{i}|i\rangle\left\langle i\left|+\sum_{i \neq j} V_{i j}(t)\right| i\right\rangle\langle j|
$$


Here the states $|i\rangle$ and $|j\rangle$ correspond to the HOMOs on different nucleobases and $\varepsilon_{i}$ is the orbital energy. In eq. 1, the effect of classical nuclear dynamics is incorporated into the time dependent couplings $V_{i j}(t)$ that summarize the effect of hundreds of possible nuclear modes, modulating the inter-base coupling.

The results of the MD/QC calculation ${ }^{[13]}$ showed that the couplings $V_{i j}(t)$ between adjacent bases undergoes fluctuations of the same order of magnitude of their average value. For example, the computed average coupling between the central pair of adenines in the double helix sequence 5'-CAGAAAAGTG-3' was $150 \mathrm{~cm}^{-1}$ and its standard deviation was $700 \mathrm{~cm}^{-1}$ (A, C, G, T denote the four standard DNA bases). The large amplitude of the computed fluctuations in the Hamiltonian makes it difficult to consider the electronic structure of DNA anyhow related to that of an idealized or 'averaged' structure. A similar observation was made also in other studies ${ }^{[14-16]}$ and it is consistent with the variability of the intermolecular coupling computed using a set of crystallographic (static) structures. ${ }^{[11]}$

The effect of these fluctuations on the electron dynamics depends on the charge transport mechanism, but there is not a consensus on the actual transport mechanism of positive charge in DNA and several contrasting hypotheses have been formulated. ${ }^{[17-20]}$ The situation is clearer in some well-defined cases extensively investigated by Giese and coworkers. ${ }^{[21]}$ In DNA sequences containing non-adjacent $\mathrm{G}$ bases, the charge transport can be seen as a series of charge transfer (CT) reactions (hopping) between a positively charged guanine $\left(\mathrm{G}^{+}\right)$and a neutral close guanine. This is reasonable since the guanine has a lower ionization potential and the aqueous environment is able to localize the charge on the nucleobase where it is more stable. ${ }^{[2]}$ The elementary process for the same sequence mentioned above can be written as

$\ldots \mathrm{AG}^{+} \mathrm{AAAAGT} \ldots \rightarrow \ldots \mathrm{AGAAAAG}^{+} \mathrm{T} \ldots$

(examples where the above elementary reaction cannot account for the observations include charge transfer through long DNA fragments not containing G or containing only GC pairs, and photoinduced charge separation processes). Assuming that a series of CT reactions actually take place, the appropriate rate constant for the process is the non-adiabatic thermal rate constant often written as: $:^{[23,24]}$

$$
k_{C T}=\frac{2 \pi}{\hbar}\left|V_{D A}\right|^{2} \rho_{F C T}
$$


where $V_{D A}$ is the effective coupling between donor (D) and acceptor (A) -the two G bases in this case-, and $\rho_{F C T}$ is the density of states weighted by the Franck-Condon factor and thermally averaged.

From the time dependent Hamiltonian of eq. 1 one can compute the (time dependent) $V_{D A}$ for the CT reaction considered above using for example the matrix partition method. ${ }^{[25]}$ Two aspects of the $V_{D A}(t)$ computed in ref. ${ }^{[13]}$ are particularly striking: (i) the standard deviation of $V_{D A}(t)$ is much larger that its average value, and (ii) the average and standard deviation of $V_{D A}(t)$ seem to be determined by a minority of time points where the $V_{D A}$ is several order of magnitudes larger than the most probable value (which is very close to zero).

Superexchange through a series of fluctuating couplings. Here we will put these observations in a more general context, valid for the electron tunneling between donor and acceptor through any one-dimensional array of $N$ pi-stacked non-bonded molecules, each interacting only with its nearest neighbors. Assuming that D and A have the same energy, well below the energy of the bridging molecules, the effective tunneling matrix element between donor an acceptor is proportional to the product of the coupling between adjacent molecules according to the well know McConnell superexchange expression: ${ }^{[26]}$

$$
V_{D A} \approx \frac{V_{D 1} V_{N A}}{\Delta E^{N}} \prod_{i=1, N-1} V_{i, i+1} .
$$

$\Delta E$ is the energy difference between the bridge sites and the $\mathrm{D}$ (or $\mathrm{A}$ ) energy. The distribution probability of $V_{D A}$ is therefore related to the distribution probability of its factors $V_{D 1}, V_{12}, V_{23}, \ldots, V_{N D}$. We have seen how, at room temperature, pi-stacked molecules not connected by a rigid molecular bridge undergo large amplitude motions that introduce a modulation to the average intermolecular coupling. For simplicity one can build a model assuming that the coupling between two adjacent molecules $V_{D 1},\left\{V_{i, i+1}\right\}, V_{N D}$ is normally distributed with the same distribution valid for any $i, i+1$ pair (see also next section for a justification of taking the normal distribution). It is not possible to express analytically the probability distribution of the product of two or more normally distributed variables (except if

\footnotetext{
\# We have seen that, in DNA, the same qualitative behavior for $V_{D A}$ is obtained using the approximate McConnell expression or the more rigorous matrix partition method.
} 
their average value is zero). ${ }^{[27]}$ The numerical evaluation of the probability distribution is done remembering that, if the variables $X$ and $Y$ are distributed according to the functions $P_{X}(x)$ and $P_{Y}(y)$, the product $Q=X Y$ is distributed according to:

$$
P_{X Y}(q)=\int_{-\infty}^{+\infty} P_{X}(x) P_{Y}\left(\frac{q}{x}\right) \frac{1}{|x|} d x
$$

[Figure 1]

In Figure 1 the probability distribution of the superexchange coupling $V_{D A}$ is shown, assuming it is determined by the product of several independent couplings normally distributed with the same average and standard deviation (see eq. 3). When the standard deviation of $V_{i, i+1}$ is of the same order of magnitude of its average, the probability distribution of $V_{D A}$ departs from the normal distribution and develops a tail for larger values of $V_{D A}$. The tail of the $V_{D A}$ distribution is larger when the product of more independent couplings is considered, and the shape of the distribution departs more radically from the normal distribution when the ratio between the standard deviation and the average value is larger than $\sim 0.5$. Above this threshold (e.g. in DNA) the most probable $V_{D A}$ value is zero and the tail of the distribution is roughly proportional to $1 /|x|^{\alpha} \quad(\alpha>1)$. This distribution seems to be typical of all tunneling phenomena through strongly fluctuating bridges and it has important consequences for the characterization of the electronic interaction is these systems. The calculation of $V_{D A}$ in the equilibrium geometry or in few 'representative' geometries is likely to give a completely misleading picture of the average coupling between donor and acceptor. In fact, the effective average coupling is determined by the tail of the distribution, i.e. by the less likely configurations. For this reason, an accurate sampling of the conformational space is an essential ingredient to characterize the coupling between configurations. This conclusion was reached independently by several groups that attempted the calculation of average couplings using conformational sampling from MD simulations including Balabin and Onuchic $^{[28]}$, Senthilkumar et al. ${ }^{[29]}$, and Skourtis et al. ${ }^{[30]}$

Organic Crystals. Discussing the dynamics of the electronic coupling between non bonded molecules, it is natural to consider also the very important case of charge transport in molecular organic crystals. ${ }^{[31]}$ These materials represent an ideal ground to study the effect of 
electronic coupling fluctuations because the other dynamical variables that can influence the charge carrier motions (conformational changes, counterions and solvent) are absent. As in the case of DNA, the nuclear dynamics of the crystal can be explored through classical MD simulations and the intermolecular electronic coupling can be evaluated for snapshots of the MD trajectory separated by fixed time intervals. An analysis of this type on anthracene, pentacene and pentacene derivatives was presented in refs. ${ }^{[32]}$ and ${ }^{[33]}$. We extend this study here to the electronic coupling fluctuations of sexithiophene, a molecule that can be considered a prototype for an entire class of organic semiconductors ${ }^{[9,34]}$ and is therefore a reference also for many theoretical studies. ${ }^{[35]}$

A supercell containing 48 molecules of sexithiophene was build replicating the crystal unit cell 2, 3 and 4 times respectively along the crystallographic axes $a, b$, and $c(a=44.70 \AA$, $b=7.81 \AA, c=6.03 \AA) .{ }^{[36]}$ The MD simulation at constant temperature ${ }^{[37]}$ was run using the MM3 force field ${ }^{[38]}$ with frozen $\mathrm{C}-\mathrm{H}$ bond distances ${ }^{[39]}$ and 2 fs integration step. Snapshots every $30 \mathrm{fs}$ were considered for the quantum chemical study. For each snapshot the HOMO orbital of 10 distinct molecules was computed with the INDO/S Hamiltonian and the coupling between 19 pairs of HOMOs was computed according to the method described in ref. ${ }^{[40]}$. The quantum chemical evaluation of the intermolecular coupling was the computationally more demanding portion of this study and limited the analyzed trajectory to 30ps (i.e. 1000 snapshots and 19000 intermolecular coupling evaluations). The quality of the INDO/S Hamiltonian for this type of calculation was repeatedly checked against more accurate DFT calculations. $^{[41]}$

\section{[Figure 2]}

In Figure 2 we showed the relative position of the molecular pairs that have the largest electronic coupling and we indicated them with $A, B, C$. The average coupling and standard deviation at $100 \mathrm{~K}$ resulting from this calculation is $-167 \pm 96,245 \pm 104$ and $189 \pm 114 \mathrm{~cm}^{-1}$ for $A, B$, and $C$ pairs respectively, while the corresponding values for the simulation at $300 \mathrm{~K}$ were $-158 \pm 149,224 \pm 161$ and $197 \pm 168 \mathrm{~cm}^{-1}$. The probability distributions of the coupling $A$ and $B$, shown in Figure 2 for simulations at $300 \mathrm{~K}$ and $100 \mathrm{~K}$, can be considered a Gaussian to a fairly good approximation (this justifies the assumption of normal distribution made in Figure 1). These results, together with previous reports, ${ }^{[32,33]}$ confirm that the coupling fluctuation is an essential feature of organic solids. It is not easy to formulate a rigorous 
transport mechanism in the presence of such fluctuation in the intermolecular electronic coupling, but it can be safely stated that the band description of the electronic structure becomes inappropriate in these cases, because nuclear thermal motions destroy the translational symmetry of the electronic Hamiltonian. In the last section, we propose a numerical approach to describe the mobility in organic semiconductors characterized by thermal electronic disorder. It is important to characterize the typical timescale of the electronic coupling modulation, which ultimately determines its role on the transport mechanism. This timescale can be evaluated by inspecting $V_{i j}(t)$ or, more rigorously, performing a Fourier transform of the autocorrelation function $\left\langle V_{i j}(t) V_{i j}(0)\right\rangle$. The greatest contribution to the coupling fluctuation derives from vibrations with energy of $\sim 50 \mathrm{~cm}^{-1}$ (corresponding to an oscillation period of $60 \mathrm{fs}) .{ }^{[32]}$ This justifies the approximation of treating the vibrations classically at room temperature $\left(\hbar \omega \square k_{b} T\right)$ and suggests that the snapshots of the MD simulation should be taken at interval shorter than $60 \mathrm{fs}$.

Generalization. In all considered systems containing non-bonded pi-stacked molecules the computed intermolecular coupling undergoes very large fluctuations at room temperature and it seems reasonable to ask weather this behavior is general or specific of the considered cases. The electron transport/transfer properties of all organic materials are dominated by the frontiers orbital of their molecular constituents. Differently from the case of simple inorganic semiconductors, like silicon or germanium, the molecular frontier orbitals have a very complex shape and contain a large number of nodal planes. The HOMO orbital of a conjugated molecule with $N \mathrm{sp}^{2}$ carbons will have approximately $N / 2$ nodal planes and it is therefore very common that the overlap between two MOs localized on two adjacent molecules results from a complicated interference between positive and negative overlaps between the two orbitals. For this reason it is very difficult to rationalize the coupling between two orbitals only from their shape and relative orientation, and it is very common that the sign of the coupling changes if one molecule is displaced with respect to the other by a fraction of an Angstrom. A couple of conjugated molecules have the maximum intermolecular coupling if they are parallel to one another and the distance between the corresponding atoms in the two molecules is at its minimum (perfect pairing). This situation corresponds to a maximum in the coupling and, therefore, small molecular displacements do not modulate effectively the 
intermolecular coupling. However, in real crystals molecules do not pack in the maximum coupling geometry and, on the contrary, the relative position of the molecules is such that the intermolecular coupling is small, and very sensitive to the exact molecular position. ${ }^{[33]}$ The subtle nature of the intermolecular interaction also explains why it has not been possible to find a simple relation between geometric and electronic structure of organic solids, notwithstanding more than three decades of systematic investigation.

\section{Electron dynamics in pi-stacked systems with fluctuations}

The electron dynamics in molecular systems is mainly determined by the relative magnitude of two energy parameters. When an excess charge (hole or electron) moves from one site (state) to another, the nuclei rearrange themselves into a new energy minimum. The energy reduction consequent to this nuclear relaxation is the reorganization energy $(\lambda)$ and it measures the tendency of the system to localize its excess charge. The electronic coupling between localized electronic states $(V)$ measures instead the tendency of the system to delocalize its electrons. Depending on the relative magnitude of electronic coupling and reorganization energy one can distinguish several limiting cases (we will describe them assuming first that $V$ is constant in time):

[Figure 3]

(i) If there are two sites for the hole (or electron) and the reorganization energy is considerably larger than the coupling between these two sites, we are in the non-adiabatic regime, by far the most common case considered in chemical quantum dynamics, and for which the theories of Marcus-Jortner-Hush can be applied. ${ }^{[23]}$ In the discussion of electron dynamics through pi-stacked systems this situation can be found for example in the charge hopping between two guanine bases separated by several non-G nucleobases in DNA, or the charge hopping between two electronic traps in an organic crystal. It is common, in these cases, to identify one effective reaction coordinate (associated with the solvent coordinate in liquid phases) and to represent the energy of the two non-interacting states along the reaction coordinate as two intersecting parabolas. The coupling $V$ between the two states causes a splitting (of $2|V|)$ between the two potential energy curves. 
(ii) Sometimes, the coupling between the two localized states is not very small, or the reorganization energy for the charge transfer reaction is modest. This is usually the case for molecules in direct contact (coupling of the order of 0.1-0.4 eV) and/or in the absence of polar solvent as in crystals. A prototypical case can be considered the charge hopping between two adjacent nucleobases in DNA or between two adjacent molecules in an organic crystal (assuming that the reorganization energy is strong enough to preserve some degree of localization $\left.^{[42]}\right)$. This is an adiabatic charge transfer reaction, i.e. the system stays always in its ground state, in contrast with the non-adiabatic case when it is very probable for the system to cross the intersection between the two parabolas without changing electronic configuration. In solid state literature the localized states of type (i) or (ii) are often called small polarons. (iii) If the reorganization energy is very weak, as for large conjugated molecules in molecular crystals (e.g. pentacene, rubrene), the charge should be delocalized. ${ }^{[43]}$ When this happens in solids the charge transport mechanism is usually said to be band-like but, as we will see, this statement is correct only at very low temperatures.

There are no neat boundaries between the three regimes, although there are traditionally different formalisms and languages used to approach each case. In particular, a great amount of work has been devoted to the coexistence of regime (ii) and (iii) in organic semiconductors. ${ }^{[44]}$ It takes in fact a small change in the parameter space to go from the charge transfer reaction regime (familiar to physical chemists) to the delocalized regime (familiar to semiconductor physicists) and it is now acknowledged that the understanding of the charge dynamics in organic semiconductors will benefit from a synthesis between the two approaches. However, the study of electron transport/transfer in the intermediate regimes is particularly challenging also in the absence of coupling fluctuation and no general model is available. It is therefore natural to study the two problems separately, i.e. considering intermediate regimes with constant coupling or a well-defined regime in the presence of coupling fluctuations. In the remainder of the section, we assume that one of the three regimes is appropriate and we consider the impact of electronic coupling fluctuation in each one of these regimes, neglecting the phenomenology for intermediate cases. Each case will involve a different set of approximations so that the accuracy of the resulting models cannot be directly compared. For reason of space we will stress the differences between regimes while we will refer the reader to the appropriate literature for other technical issues specific of each regime. 


\section{3.i Charge transfer through fluctuating bridge in the non adiabatic limit.}

In the non-adiabatic limit and if the coupling between donor and acceptor states can be considered constant (Condon approximation) the appropriate expression of the rate is given by eq. 2. Several researchers revisited the derivation of the non-adiabatic CT rate expression allowing for the presence of a time dependent $V_{D A}(t)$ coupling (non-Condon effects) and the readers is referred to these papers for a more complete overview of the current research. ${ }^{\text {[45-49] }}$ Following for example ref. ${ }^{[47]}$, the rate constant from a particular vibrational state $v$ of the donor to the vibrational manifold of the acceptor is given by,

$$
k_{v}=\int_{-\infty}^{+\infty} J\left(E-E_{v}\right) \rho_{F C v}(E) d E
$$

where the spectral density $J$ contains the information on the fluctuation of the coupling $V_{D A}$ and it is defined as a Fourier transform of the autocorrelation function of $V_{D A}(t)$ :

$$
J(E)=\frac{1}{\hbar^{2}} \int_{-\infty}^{+\infty}\left\langle V_{D A}(t) V_{D A}(0)\right\rangle e^{(i / \hbar) E t} d t
$$

It is easy to verify that, if $V_{D A}$ is constant in time, eq. 6 gives $J(E)=2 \pi \delta(E) / \hbar$, which leads to the conventional golden rule equation (eq.2). An appealing property of this formalism is that it is possible to obtain a series expansion of the rate constant

$$
k=k^{(0)}+k^{(1)}+k^{(2)}+\ldots,
$$

where the leading term coincides with the rate constant in the static limit

$$
k^{(0)}=\frac{2 \pi}{\hbar}\left\langle V_{D A}^{2}\right\rangle \rho_{F C T}
$$

and the remaining terms $k^{(1)}, k^{(2)}$, etc..., are the corrections due to the fluctuations of the coupling. If the nuclear modes are treated classically (numerical simulations show that only low frequency modes modulate the electronic coupling), the first non zero correction is

$$
k^{(2)}=\frac{\pi \hbar}{\tau_{C}{ }^{2}}\left\langle\left(V_{D A}-\left\langle V_{D A}\right\rangle\right)^{2}\right\rangle \rho_{F C T}^{\prime \prime}
$$

Where $\tau_{C}$ is the characteristic timescale for the fluctuation of the coupling and $\rho_{F C T}^{\prime \prime}$ is the second derivative with respect to the energy of $\rho_{F C T}$. It is therefore possible to assess if it is 
meaningful to truncate the series expansion to the first term $k^{(0)}$, evaluating the magnitude of the correction in eq. 9. Considering explicitly the expression for $\rho_{F C T}$ derived by Marcus one finds:

$$
\begin{aligned}
& k^{(0)}=\frac{\left\langle V_{D A}^{2}\right\rangle}{\hbar} \sqrt{\frac{\pi}{\lambda k_{B} T}} \exp \left(-\frac{\left(\lambda+\Delta E^{0}\right)^{2}}{4 \lambda k_{B} T}\right) \\
& k^{(2)}=k^{(0)} 2 \frac{\hbar^{2}}{\tau_{C}^{2}}\left[\frac{\left(\lambda+\Delta E^{0}\right)^{2}-2 \lambda k_{B} T}{\left(4 \lambda k_{B} T\right)^{2}}\right]\left(1-\frac{\left\langle V_{D A}\right\rangle^{2}}{\left\langle V_{D A}{ }^{2}\right\rangle}\right)
\end{aligned}
$$

Several authors ${ }^{[16,29,30,40]}$ have evaluated $k^{(0)}$ and $k^{(2)}$ from eq. 10-11 and found that the correction is typically very small $\left(k^{(2)}<0.1 k^{(0)}\right)$. Therefore, the intuitive idea of substituting the coupling in eq. 2 with its average value (eq. 8) is acceptable most of the times, and no major macroscopic deviation of the expected temperature dependence of the rate constant is expected for the presence of fluctuations of $V_{D A}$. However, it is important to point out that, because of the very broad distribution of $V_{D A}$ that is found so frequently (see discussion in the previous section), it is very difficult to compute accurately $\left\langle V_{D A}^{2}\right\rangle$ since the averaging requires an extensive exploration of the system conformational space.

\section{3.ii Charge transfer in the presence of fluctuating coupling in the adiabatic limit.}

The adiabatic limit is not very often considered in the literature of charge transfer because it is traditionally associated to the study of ground state reaction dynamics, which make use of a quite different set of methods and approximations. However, the adiabatic limit can be common when discussing the $\mathrm{CT}$ reaction between adjacent pi-stacked molecules, since, in this case, the intermolecular coupling (typically between 80 and $2000 \mathrm{~cm}^{-1}$ ) can be strong enough to split the potential energy surface of the two electronic states (see Figure 3(ii)), so that the reaction proceeds in the ground state. In DNA for example, it is probable that the adiabatic and non-adiabatic charge transfer models are appropriate for the CT reaction between adjacent and non-adjacent nucleobases respectively. 
The effect of electron coupling fluctuations in adiabatic CT is considerably different. Since the splitting between the two states is twice $V_{D A}(t) \stackrel{\S}{\S}$ indicating with $\delta V(t)$ the difference $\delta V(t)=V_{D A}(t)-\left\langle V_{D A}(t)\right\rangle$, we find that the activation energy is modulated in time by $E_{a}(t)=\left\langle E_{a}\right\rangle-\delta V(t)$ (it must be $|\delta V(t)|<\left\langle E_{a}\right\rangle$ to be in this regime). One can therefore write an Arrhenius type expression of the rate constant with the activation energy being modulated by the fluctuating intermolecular coupling:

$$
k(t)=A \exp \left(-\left(\left\langle E_{a}\right\rangle-\delta V(t)\right) / k_{B} T\right)
$$

The previous equation is valid if the modulation $\delta V(t)$ is slow compared to the frequency modes along the reaction coordinate. This can be the case for reactions involving adjacent conjugated molecules, where the reorganization modes (reaction path) are a combination of fast C-C stretching modes, while the modes inducing the fluctuation in the electronic coupling are low frequency motions of one molecule with respect to the other. Under this condition, it makes sense to define an averaged rate constant as:

$$
k^{C T} \equiv\langle k(t)\rangle=A\left\langle\exp \left(-\frac{E_{a}(t)}{k_{B} T}\right)\right\rangle \approx A \exp \left(-\frac{\left\langle E_{a}\right\rangle}{k_{B} T}\right) \exp \left(\frac{\left\langle\delta V^{2}\right\rangle}{2\left(k_{B} T\right)^{2}}\right),
$$

where the last term was obtained using the cumulant expansion truncated to the second term:

$$
\langle\exp (\alpha x)\rangle=\exp \left[\alpha\langle x\rangle+\frac{1}{2} \alpha^{2}\left(\left\langle x^{2}\right\rangle-\langle x\rangle^{2}\right)+\ldots\right] \text {. }
$$

If we define, as before, the zero order rate constant as the one in the absence of fluctuations $k^{(0)}=A \exp \left(-\left\langle E_{a}\right\rangle / k_{B} T\right)$, the effect of the fluctuation can be seen as a correcting factor to $k^{(0)}$ and Eq. 13 can be rewritten as

$$
k^{C T} \equiv k^{(0)} \exp \left(\frac{\left\langle\delta V^{2}\right\rangle}{2\left(k_{B} T\right)^{2}}\right) .
$$

This expression, like eqs. 7-9, relates the rate constant in the presence of coupling with the value of the rate constant in the frozen configuration (eq. 15 is less general than eq/7-9 being valid only for 'slow' fluctuations). It is immediately clear that the effect can be substantial only if the amplitude of the fluctuation is large compared with $k_{B} T$. Both in eqs. 15 and 9 , the

\footnotetext{
$\S^{\S}$ To simplify the notation we have assumed $V_{D A}>0$, but the same final result is obtained if $V_{D A}<0$.
} 
correction due to the coupling fluctuation leads to an increase of the effective rate with respect to an 'averaged' situation.

We can build a small model system to predict the temperature dependence of the correcting factor, assuming that the coupling fluctuation is only due to a single harmonic nuclear degree of freedom $q$, which modulates $V$ according to:

$$
V(t)=\langle V\rangle+\alpha q(t)
$$

Since $\left\langle\delta V^{2}\right\rangle=\alpha^{2}\left\langle q^{2}\right\rangle$ and, for the equipartition principle, $\left\langle q^{2}\right\rangle=k_{B} T / m \omega^{2}$ (being $m$ and $\omega$ the mass and frequency of the oscillator), we get

$$
k^{C T}=A \exp \left[-\frac{1}{k_{B} T}\left(\left\langle E_{a}\right\rangle-\frac{\alpha^{2}}{2 m \omega^{2}}\right)\right]
$$

Eq. 17 implies that the main effect of fluctuations is a reduction of the apparent activation energy. However, it seems difficult to find a fingerprint of the fluctuation in measurements of the rate constant, although for the prediction of rate constants (as in the non-adiabatic case) it is again necessary to perform a good exploration of the conformational space to compute $\left\langle\delta V^{2}\right\rangle$ accurately.

\section{3.iii Charge transport in the "delocalized" case with fluctuating coupling}

The consequence of electronic coupling fluctuation on the electron dynamics is particularly important in organic solids. In the solid state, the reorganization energy is largely due to intramolecular vibrational modes while, in solution, the effect of the solvent is equivalently or more important. The intramolecular reorganization energy decreases monotonically increasing the size of conjugated molecules, ${ }^{[50]}$ while the typical intermolecular coupling (that do not follow a simple pattern) is of similar magnitude for any pairs of molecules in contact. For larger molecules the reorganization energy is so small (e.g. $0.09 \mathrm{eV}$ for pentacene) that it is not able to localize the charge carriers (holes in the case of pentacene). We are in case (iii) pictured in Figure 3: the electron should be delocalized and the charge transport described by a band model. In a band transport model, carriers delocalized over many unit cells are described by wavepackets with a given crystal momentum $k$ and they are scattered to a new state $k^{\prime}$ by the interaction with phonons or impurities. It is possible to build a band transport model whose parameters are adjusted to reproduce the transport properties in organic 
materials. ${ }^{[51]}$ However, if the fluctuation of intermolecular coupling is very strong, the electronic band structure (at $0 \mathrm{~K}$ ) ceases to be a good description of the electronic states at room temperature, where the translational symmetry of the electronic Hamiltonian is completely broken by the dynamic disorder. It is easy to verify that, in the presence of such disorder, the charge carriers become localized over few unit cells. The dynamics of the charge carriers becomes intimately bound to the dynamic of the nuclei, since it is through the nuclear motions that the localized states are mixed one another and the carriers propagate through the lattice. It is essential to differentiate the localization due to the dynamic disorder (which is important when the reorganization energy is low and insufficient to localize the charge) and the localization due to the small polaron formation (which is found when the reorganization energy is sufficiently high).

The charge transport mechanism based on the dynamic localization has not been studied extensively in solid state physics and no analytical treatment has been attempted so far. The following simplified model can be used to study numerically the basic features of the charge carrier motions in systems with dynamic disorder (see Figure $4 a):{ }^{[52]}$

$$
H=\sum_{j}\left(-\tau+\alpha\left(u_{j+1}-u_{j}\right)\right)(|j\rangle\langle j+1|+| j+1\rangle\langle j|)+\sum_{j} \frac{1}{2} m \dot{u}_{j}^{2}+\sum_{j} \frac{1}{2} K u_{j}^{2}
$$

[Figure 4]

A 1-dimensional array of molecules is considered, with a single nuclear mode $u_{j}$ (harmonic, with mass $m$ and force constant $K$ ) and a single orbital $|j\rangle$ per molecule. The coupling between two consecutive orbitals $|j\rangle$ and $|j+1\rangle$ is modulated by the classical nuclear modes according to the matrix element $-\tau+\alpha\left(u_{j+1}-u_{j}\right)$. The transport regime resulting from eq. 18 depends on the set of chosen parameters. A typical set based on our recent calculation on the pentacene solid is given by $K=14500 \mathrm{amu} \mathrm{ps}{ }^{-2}, \tau=300 \mathrm{~cm}^{-1}, \alpha=995 \mathrm{~cm}^{-1} / \AA, m=250 \mathrm{amu}$. We note that the model in eq. 18 implies a Gaussian distribution of the intermolecular coupling at any given temperature deriving from the Gaussian distribution of the displacements $u_{j}$.

Details on the numerical integration of eq. 18 are given in ref. ${ }^{[52]}$ and a similar model is discussed in the context of DNA transport by Senthilkumar et al. ${ }^{[29]}$. The initial wavefunction, taken to be one of the Hamiltonian eigenfuctions at $t=0$, is localized on few 
molecular sites (with the considered parameter set at room temperature). Because of the dynamic coupling between adjacent molecules, the initial wavefunction spreads over the lattice and its time dependent diffusion can be monitored evaluating the quantity

$$
R_{n}^{2}(t) \equiv\left\langle\psi_{n}(t)\left|r^{2}\right| \psi_{n}(t)\right\rangle-\left\langle\psi_{n}(t)|r| \psi_{n}(t)\right\rangle^{2}
$$

Many trajectories can be computed starting from different initial wavefunctions $\psi_{n}(t=0)$ (and energy $E_{n}$ ) and the average $\left\langle R^{2}(t)\right\rangle$ can be computed by Boltzman averaging the quantity $R_{n}^{2}(t)$ over different trajectories. Figure $4 \mathrm{~b}$ shows the behavior of $R_{n}^{2}(t)$ for selected starting wavefunctions $\psi_{n}(t=0)$ and the average $\left\langle R^{2}(t)\right\rangle$ computed from 125 trajectories at $300 \mathrm{~K}$. As discussed elsewhere, ${ }^{[52]}$ this model describes quite accurately the main features of the charge transport in crystals of large conjugated molecules but it is probably too simple to be considered valid for a generic molecular semiconductor. First, one needs to include the effect of dephasing on the evolution of the wavefunctions that now evolves only coherently in the lattice (the current model is valid if the dephasing time is slower than the intermolecular vibrations). Secondly, it is also important to incorporate the effect of reorganization energy that is neglected in eq 18 (this is expected to be important for smaller conjugated molecules like naphthalene). ${ }^{[43]}$

Despite its limitations, the model in eq. 18 illustrates quite clearly an important point: the fluctuations of the intermolecular coupling are the factor that determines (and limit) the mobility in organic semiconductors, i.e. they are not a correction on the charge dynamics observed in their absence (as in the case of non-adiabatic and adiabatic CT reactions).

\section{Conclusion}

We have provided further evidence that the electron coupling between pi-stacked molecules presents typically strong fluctuations due to thermal motions. The effects of these fluctuations are different and depend of the regime of charge transport/transfer that is being considered. Usually, the stronger is the charge localization due to the reorganization energy the weaker is the effect of the coupling fluctuation on the electron dynamics. In the case of non-adiabatic 
CT reactions (strong reorganization energy and weak coupling), the fluctuations cause a small additive correction to the expression of the rate constant, which can be accurately evaluated using the squared averaged electronic coupling between donor and acceptor. In the case of adiabatic CT reactions (reorganization energy strong enough to localize the charge but comparable with the donor-acceptor coupling) the effect is larger, and the correction due to the fluctuation of the coupling appears as a multiplicative correction to the rate constant. For both cases where the reorganization energy is sufficient to localize the charge, the presence of fluctuations has a greater impact on the computational modeling than on the analysis of the experimental data. In fact, while it is difficult to find evidence of the coupling fluctuations from the experiments, the computation of the electronic coupling needed to interpret the experiments requires an accurate average over the broad region of conformational space explored by the system. A much stronger effect of the fluctuation of the electronic coupling is found when the reorganization energy is small compared to the intermolecular coupling and the conventional models would suggest that the charge carriers are delocalized. In this case, the fluctuation causes dynamic localization of the charge and determines the transport mechanism instead of introducing a small correction.

The present description of the effects of coupling fluctuation has neglected the existence of intermediate regimes and focused mainly on highly idealized systems. Currently, in the absence of simple phenomenological models for intermediate regimes with fluctuating electronic coupling, these complicated cases can be studied only through fully numerical simulations involving a suitable extension of the Hamiltonian in eq. 18. Additional theoretical and computational work is necessary to translate these ideas into a practical set of computational tools for the prediction of the charge dynamics in materials containing nonbonded pi-stacked molecules.

I am grateful to Prof. G. Orlandi for his help in the study of the charge transport mechanism in organic materials and to Prof. A. Nitzan and Prof. M. Ratner for illuminating discussions on the theory of electron tunneling through fluctuating bridges. This work is supported by the Research Council UK. 


\section{References:}

[1] C. D. Dimitrakopoulos, P. R. L. Malenfant. Organic thin film transistors for large area electronics. Adv. Mater., 14, 99 (2002).

[2] O. D. Jurchescu, J. Baas, T. T. M. Palstra. Effect of impurities on the mobility of single crystal pentacene. Appl. Phys. Lett., 84, 3061 (2004).

[3] V. Podzorov, E. Menard, J. A. Rogers, M. E. Gershenson. Hall effect in the accumulation layers on the surface of organic semiconductors. Phys. Rev. Lett., 95 (2005).

[4] D. Porath, G. Cuniberti, R. Di Felice, in Long-Range Charge Transfer in DNA Ii, Vol. 237, 2004, pp. 183.

[5] R. G. Endres, D. L. Cox, R. R. P. Singh. Colloquium: The quest for high-conductance DNA. Rev. Mod. Physics, 76, 195 (2004).

[6] Y. A. Berlin, I. V. Kurnikov, D. Beratan, M. A. Ratner, A. L. Burin, in Long-Range Charge Transfer in DNA Ii, Vol. 237, 2004, pp. 1.

[7] A. Fechtenkotter, K. Saalwachter, M. A. Harbison, K. Mullen, H. W. Spiess. Highly ordered columnar structures from hexa-peri-hexabenzocoronenes - Synthesis, X-ray diffraction, and solid-state heteronuclear multiple-quantum NMR investigations. Angew. Chem.-Int. Edit., 38, 3039 (1999).

[8] A. M. van de Craats, N. Stutzmann, O. Bunk, M. M. Nielsen, M. Watson, K. Mullen, H. D. Chanzy, H. Sirringhaus, R. H. Friend. Meso-epitaxial solution-growth of selforganizing discotic liquid-crystalline semiconductors. Adv. Mater., 15, 495 (2003).

[9] H. Sirringhaus. Device physics of Solution-processed organic field-effect transistors. Adv. Mater., 17, 2411 (2005).

[10] J. L. Bredas, J. P. Calbert, D. A. Da Silva Filho, J. Cornil. Organic semiconductors: a theoretical characterization of the basic parameters governing charge transport. Proc. Natl. Acad. Sci. U. S. A., 99, 5804 (2002).

[11] A. Troisi, G. Orlandi. The hole transfer in DNA: calculation of electron coupling between close bases. Chem. Phys. Lett., 344, 509 (2001).

[12] P. M. Kazmaier, R. Hoffmann. A Theoretical-Study of Crystallochromy - Quantum Interference Effects in the Spectra of Perylene Pigments. J. Am. Chem. Soc., 116, 9684 (1994).

[13] A. Troisi, G. Orlandi. Hole migration in DNA: a theoretical analysis of the role of structural fluctuations. J. Phys. Chem. B, 106, 2093 (2002).

[14] A. A. Voityuk, K. Siriwong, N. Rosch. Charge transfer in DNA. Sensitivity of electronic couplings to conformational changes. Physical Chemistry Chemical Physics, 3, 5421 (2001).

[15] A. A. Voityuk, K. Siriwong, N. Rosch. Environmental fluctuations facilitate electronhole transfer from guanine to adenine in DNA pi stacks. Angew. Chem.-Int. Edit., 43, 624 (2004).

[16] T. Cramer, T. Steinbrecher, A. Labahn. Static and dynamic aspects of DNA charge transfer: a theoretical perspective. Physical Chemistry Chemical Physics, 7, 4039 (2005).

[17] E. M. Conwell. Charge transport in DNA in solution: The role of polarons. Proc. Natl. Acad. Sci. U. S. A., 102, 8795 (2005). 
[18] M. A. O'Neill, J. K. Barton. DNA charge transport: Conformationally gated hopping through stacked domains. J. Am. Chem. Soc., 126, 11471 (2004).

[19] Y. A. Berlin, A. L. Burin, M. A. Ratner. Elementary steps for charge transport in DNA: thermal activation vs. tunneling. Chem. Phys., 275, 61 (2002).

[20] A. Joy, G. B. Schuster. Long-range radical cation migration in DNA: Investigation of the mechanism. Chemical Communications, 2778 (2005).

[21] B. Giese, S. Wessely, M. Spormann, U. Lindemann, E. Meggers, M. E. MichelBeyerle. On the mechanism of long-range electron transfer through DNA. Angew. Chem.-Int. Edit., 38, 996 (1999).

[22] I. V. Kurnikov, G. S. M. Tong, M. Madrid, D. N. Beratan. Hole size and energetics in double helical DNA: Competition between quantum delocalization and solvation localization. J. Phys. Chem. B, 106, 7 (2002).

[23] M. A. Ratner. Bridge-Assisted Electron-Transfer - Effective Electronic Coupling. $J$. Phys. Chem., 94, 4877 (1990).

[24] M. D. Newton, in Electron Transfer-from Isolated Molecules to Biomolecules, Pt 1, Vol. 106, 1999, pp. 303.

[25] S. S. Skourtis, D. N. Beratan, in Electron Transfer-from Isolated Molecules to Biomolecules, Pt 1, Vol. 106, 1999, pp. 377.

[26] A. Nitzan. Electron Transmission Through Molecules and Molecular Interfaces. Annu. Rev. Phys. Chem., 52, 681 (2001).

[27] J. K. Patel, C. B. Read, Handbook of the Normal Distribution, Dekker, New York, 1982.

[28] I. A. Balabin, J. N. Onuchic. Dynamically controlled protein tunneling paths in photosynthetic reaction centers. Science, 290, 114 (2000).

[29] K. Senthilkumar, F. C. Grozema, C. F. Guerra, F. M. Bickelhaupt, F. D. Lewis, Y. A. Berlin, M. A. Ratner, L. D. A. Siebbeles. Absolute rates of hole transfer in DNA. J. Am. Chem. Soc., 127, 14894 (2005).

[30] S. S. Skourtis, I. A. Balabin, T. Kawatsu, D. N. Beratan. Protein dynamics and electron transfer: Electronic decoherence and non-Condon effects. Proc. Natl. Acad. Sci. U. S. A., 102, 3552 (2005).

[31] M. Pope, C. E. Swenberg, Electronic Processes in Organic Crystals and Polymers, 2nd ed., Oxford University Press, New York, 1999.

[32] A. Troisi, G. Orlandi. Dynamics of the intermolecular transfer integral in crystalline organic semiconductors. J. Phys. Chem. A, 110, 4065 (2006).

[33] A. Troisi, G. Orlandi, J. E. Anthony. Electronic Interactions and Thermal Disorder in Molecular Crystals Containing Cofacial Pentacene Units. Chem. Mat., 17, 5024 (2005).

[34] A. Facchetti, M. Mushrush, M. H. Yoon, G. R. Hutchison, M. A. Ratner, T. J. Marks. Building blocks for n-type molecular and polymeric electronics. Perfluoroalkyl-versus alkyl-functionalized oligothiophenes (nT; $n=2-6)$. Systematics of thin film microstructure, semiconductor performance, and modeling of majority charge injection in field-effect transistors. J. Am. Chem. Soc., 126, 13859 (2004).

[35] J. Cornil, J. P. Calbert, D. Beljonne, R. Silbey, J. L. Bredas. Charge transport versus optical properties in semiconducting crystalline organic thin films. Adv. Mater., 12, 978 (2000). 
[36] G. Horowitz, B. Bachet, A. Yassar, P. Lang, F. Demanze, J. L. Fave, F. Garnier. Growth and Characterization of Sexithiophene Single-Crystals. Chem. Mat., 7, 1337 (1995).

[37] H. J. C. Berendsen, J. P. M. Postma, W. F. van Gunsteren, A. di Nola, J. R. Haak. J. Chem. Phys., 81, 3684 (1984).

[38] N. L. Allinger, F. Li, L. Yan, J. C. Tai. Molecular mechanics (MM3) calculations on conjugated hydrocarbons. J. Comput. Chem., 11, 868 (1990).

[39] H. C. Andersen. RATTLE - A velocity version of the chake algorithm for moleculardynamics calculations. J. Comput. Phys. , 52, 24 (1983).

[40] A. Troisi, M. A. Ratner, M. B. Zimmt. Dynamic nature of the intramolecular electronic coupling mediated by a solvent molecule: A computational study. J. Am. Chem. Soc., 126, 2215 (2004).

[41] J. L. Bredas, D. Beljonne, V. Coropceanu, J. Cornil. Charge-transfer and energytransfer processes in pi-conjugated oligomers and polymers: A molecular picture. Chem. Rev., 104, 4971 (2004).

[42] A. A. Voityuk. Charge transfer in DNA: Hole charge is confined to a single base pair due to solvation effects. J. Chem. Phys., 122 (2005).

[43] K. Hannewald, P. A. Bobbert. Ab initio theory of charge-carrier conduction in ultrapure organic crystals. Appl. Phys. Lett., 85, 1535 (2004).

[44] S. Fratini, S. Ciuchi. Dynamical mean-field theory of transport of small polarons. Phys. Rev. Lett., 91, 256403 (2003).

[45] J. Tang. Effects of a Fluctuating Electronic Coupling Matrix Element on ElectronTransfer Rate. J. Chem. Phys., 98, 6263 (1993).

[46] E. S. Medvedev, A. A. Stuchebrukhov. Breakdown of the Born-OppenheimerCondon-Marcus approximation in long distance electron transfer. Chem. Phys., 296, 181 (2004).

[47] A. Troisi, A. Nitzan, M. A. Ratner. A rate constant expression for charge transfer through fluctuating bridges. J. Chem. Phys., 119, 5782 (2003).

[48] S. J. Jang, M. D. Newton. Theory of torsional non-Condon electron transfer: A generalized spin-boson Hamiltonian and its nonadiabatic limit solution. J. Chem. Phys., 122 (2005).

[49] A. Teklos, S. S. Skourtis. Electron transfer through time dependent bridges: Differences between Franck-Condon and Born-Oppenheimer breakdown. Chem. Phys., 319, 52 (2005).

[50] V. Coropceanu, M. Malagoli, D. A. da Silva Filho, N. E. Gruhn, T. G. Bill, J. L. Bredas. Hole- and electron-vibrational couplings in oligoacene crystals: intramolecular contributions. Phys. Rev. Lett., 89, 275503 (2002).

[51] V. M. Kenkre, J. D. Andersen, D. H. Dunlap, C. B. Duke. Unified Theory of the mobilities of photoinjected electrons in naphthalene. Phys. Rev. Lett., 62, 1165 (1989).

[52] A. Troisi, G. Orlandi. Charge-transport regime of crystalline organic semiconductors: Diffusion limited by thermal off-diagonal electronic disorder. Phys. Rev. Lett., 96 (2006). 

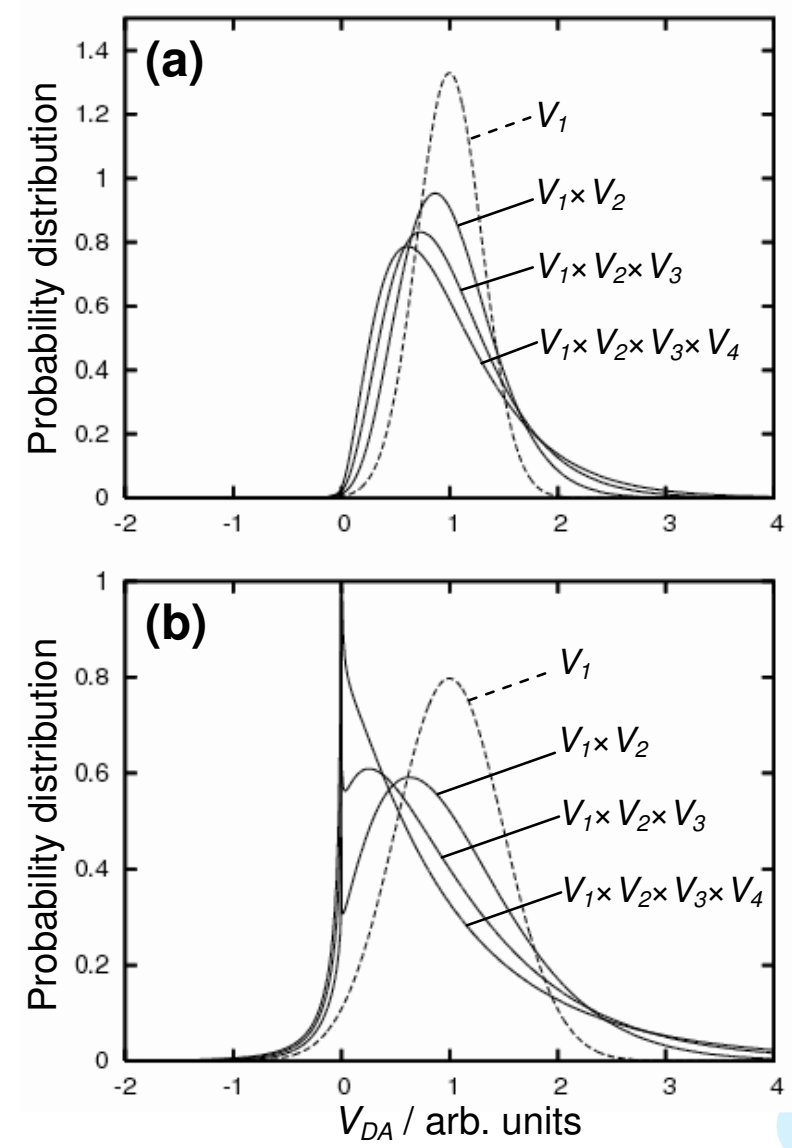

Figure 1. Probability distribution of the superexchange tunneling matrix element $V_{D A}$ assuming it is proportional to the product of 2, 3 or 4 uncorrelated normally distributed individual couplings with average 1 (arbitrary units) and variance 0.3 (a) and 0.5 (b). The dashed line represents the distribution of each individual coupling. The distribution of $V_{D A}$ acquires a tail for high coupling value. If the standard deviation is sufficiently large with respect to the average value, the most probable $V_{D A}$ value becomes zero. 

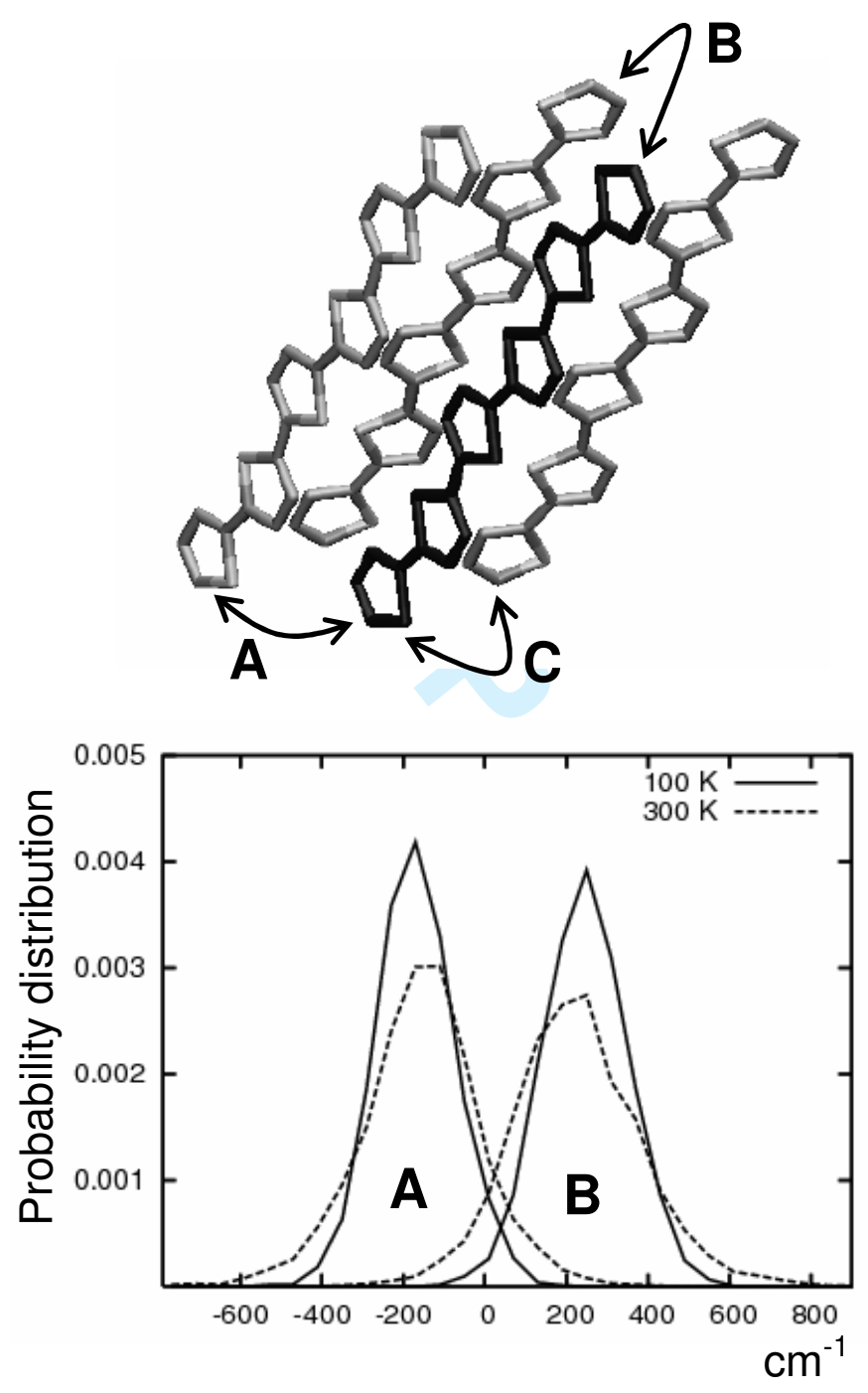

Figure 2. (top) Arrangement of sexithiophene molecules in the crystal and indication of the most strongly interacting pairs $A, B, C$. (bottom) Probability distribution at $100 \mathrm{~K}$ and $300 \mathrm{~K}$ of the electronic couplings between the HOMO orbitals of the A and B pair ( $\mathrm{C}$ is omitted for clarity). 
Figure 3. The three idealized charge transfer/transport regimes encountered in systems containing pi-stacking interaction (see text for examples). The two displaced parabolas represent the potential energy surface of the states with the charge localized on two different molecules. From left to right the reorganization energy $\lambda$ is kept constant while the coupling $V$ increases. With strong coupling (regime iii) the charge is delocalized and a CT rate cannot be defined. Fluctuations of $V$ have different effects in the three cases. 

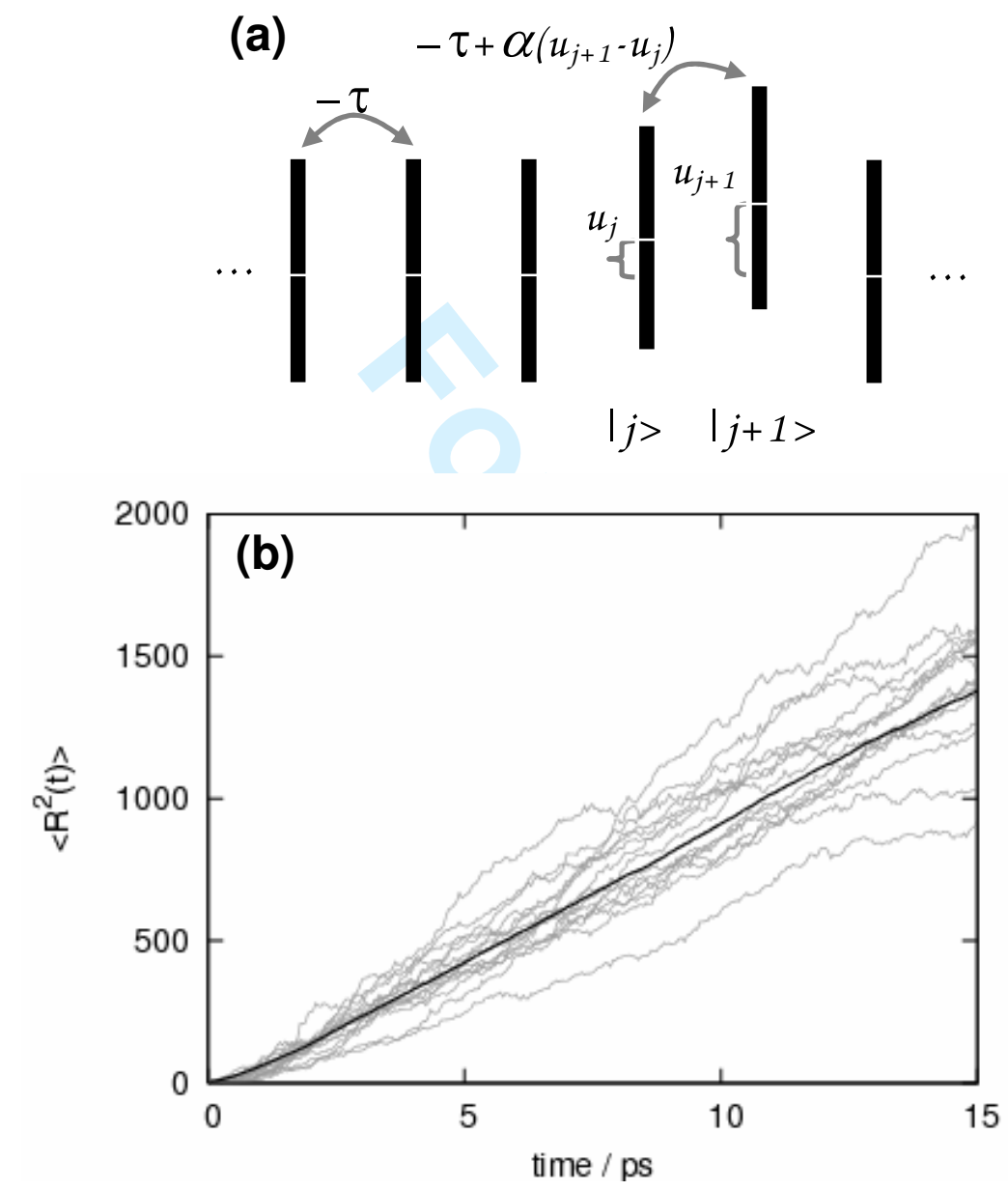

Figure 4. (a) Schematics of the model Hamiltonian of eq. 18: molecules in a 1-dimensional stack interact with their nearest neighbors through a coupling $-\tau$ modulated by the displacements $u_{j}$ of each molecule from its equilibrium position. (b) Time evolution of the spread of the wavefunction (defined in eq. 19). Gray lines refer to 15 independent trajectories, while the black line corresponds to the average over 125 trajectories at $300 \mathrm{~K}$. 